\title{
Report on the activities of the Hellenic Committee of Trainees in Obstetrics and Gynecology representatives for the year 2018 and planning of future activities
}

\author{
Anastasios Potiris ${ }^{1}$, Athanasios Douskos ${ }^{2}$
}

'Second Department of Obstetrics and Gynecology, Medical School of the National and Kapodistrian University of Athens, Aretaieion University Hospital, Greece

${ }^{2}$ First Department of Obstetrics and Gynecology, Medical School of the National and Kapodistrian University of Athens, Alexandra General Hospital, Greece

First of all, via this letter, we would like to inform you about the actions in which we participated in 2018. After the establishment of the Hellenic Committee of Trainees in Obstetrics and Gynecology, we took part in the annual Scientific Meeting and Council of the European Network of Trainees in Obstetrics and Gynaecology (ENTOG) as well as in the 26th European Congress of the European Board and College of Obstetrics and Gynecology (EBCOG), both of which were successfully held in Paris and you have received our preceding reports.

After a smooth collaboration with the project manager of PACT, a presentation on the need for a single training program in Europe and the role of PACT was given through a video-recorded speech in Brussels by Dr Athanasios Douskos.

Consequently, during the 14th Hellenic Congress of Obstetrics and Gynecology, we organised the first session dedicated to Trainees with the participation of ENTOGs President and PACT Project Manager. This session was chaired by HSOGs Special Secretary and attended by EBCOGs President - elect.

Within the framework of this session, representa- tive Dr. Athanasios Douskos presented the efforts for completing the PACT translation intoour native language, followed by the presentation of Dr. Anastasios Potiris which was based on our future goal to claim the 2021 ENTOG Exchange Program with the name "Greece 2021".

Ultimately, for the first time, our Committee was represented at the WATOG (World Association of Trainees in Obstetrics and Gynecology) and its General Assembly, which was held in Rio, Brazil. Representative Dr. Anastasios Potiris participated and represented our Committee as part of the ENTOG Delegationin the General Assembly. During his 10minute statement, he referred to the work of the Committee, the PACT and the goal of "Greece 2021".

By completing one year of operation, ourCommittee is now active in full scale at the level of trainees, with active presence and participation.Through our actions and efforts, we have re-established our relationship with Europe and the corresponding European Network as well as the aforementioned World Association. At the same time, our Committee is one of the most active National Committees. 
For the upcoming months, we aim to complete the translation of the EBCOG - PACT, organize our second trainee-based session at our HSOG Annual Meeting and ultimately to support our nomination for the ENTOG Exchange Program, Scientific Meeting and Council of the year 2021, namely "Greece 2021".

We are proud of being part of the HSOG family and we are looking forward to your support for our forthcoming plans.

\author{
Yours sincerely, \\ Anastasios Potiris \\ Athanasios Douskos
}

